\title{
Capacidade de exercício e função pulmonar de escolares com história de displasia broncopulmonar: revisão sistemática
}

\section{Exercise capacity and pulmonary function of school age children with a history of bronchopulmonary dysplasia: a systematic review}

\author{
Janaina C. Scalco ${ }^{1}$, Daiane L. de Medeiros², Camila I. S. Schivinski ${ }^{3}$
}

\begin{abstract}
RESUMO
Introdução: a displasia broncopulmonar (DBP) é uma doença respiratória crônica decorrente da necessidade de oxigênio e/ou ventilação mecânica no período neonatal. A lesão pulmonar decorrente da DBP pode levar a alterações na função pulmonar, com limitação do fluxo e aumento da resistência das vias aéreas, que perduram durante a infância e adolescência. Objetivo: realizar uma revisão sistemática de estudos que avaliaram a função pulmonar e a capacidade de exercício de escolares com DBP. Método: realizadas buscas sistematizadas em três bases de dados eletrônicas SciELO, PEDro e MEDLINE via Ovid, sendo selecionados artigos publicados até janeiro de 2014 que avaliaram tanto a função pulmonar como a capacidade de exercício em escolares com DBP. Resultados: através das estratégias de busca foram encontrados 376 títulos relacionados ao tema. Após análise dos critérios de inclusão, apenas cinco trabalhos foram selecionados para compor amostra final. Conclusões: crianças com história de DBP apresentam variável obstrução ao fluxo aéreo em idade escolar e podem atingir menores valores de consumo máximo de oxigênio, volume minuto e volume corrente durante o exercicio. Ainda assim, a função pulmonar parece não influenciar a capacidade de exercício nesta população.
\end{abstract}

Palavras-chave: Displasia Broncopulmonar. Doenças Pulmonares. Testes de Função Respiratória. Tolerância ao Exercício. Criança. Adolescente.

\begin{abstract}
Bronchopulmonary dysplasia (BPD) is a chronic respiratory disease accused by the need of oxygen and / or mechanical ventilation in the neonatal period. The resulting lung injury of BPD may lead to changes in lung function, with flow limitation and increased airway resistance, which may persist throughout childhood and adolescence to adulthood. Objective: conduct a systematic review studies that evaluated lung function and exercise capacity in school-age children with BPD. Method: we performed systematic searches of three electronic databases Scielo, PEDro and MEDLINE Ovid, being selected articles published up to
\end{abstract}

1. Fisioterapeuta Especialista em Fisioterapia Hospitalar, Mestranda do Programa de Pós-graduação em Fisioterapia da Universidade do Estado de Santa Catarina (UDESC) Florianópolis / SC / Brasil.

2. Fisioterapeuta, Mestre em Fisioterapia - UDESC

3. Professora Doutora do Departamento de Fisioterapia da UDESC.
Correspondencia Camila I. S. Schivinski Universidade do Estado de Santa Catarina (UDESC) Centro de Ciências da Saúde e do Esporte (CEFID).

Artigo recebido em 07/08/2014 Aprovado para publicação em 18/03/2015 
january 2014. That assessed pulmonary function and exercise capacity in school-age children with bronchopulmonary dysplasia. Results: through the search strategies 376 titles related to the topic were found, after analysis of the inclusion criteria, only five were selected to compose the final sample. Conclusion: children with a history of BPD have variable airflow obstruction at school age and can reach lower maximal values of oxygen, minute volume and tidal volume during exercise. Still, there is no influence lung function on exercise capacity in this population.

Keywords: Bronchopulmonary Dysplasia. Lung Diseases. Respiratory Function Tests. Exercise Tolerance. Child. Adolescent.

\section{Introdução}

Avanços nos cuidados neonatais relacionado ao desenvolvimento de tratamentos que minimizam a lesão pulmonar como: uso de corticosteroides prénatais, a introdução do surfactante exógeno pós-natal e técnicas ventilatórias protetoras reduziram os índices de mortalidade em recém-nascidos pré-termos (RNPT) ${ }^{1,2,3}$ No entanto, os índices de morbidade respiratória nessa população não diminuíram. ${ }^{4}$

A displasia broncopulmonar (DBP) é considerada a principal causa de complicação respiratória em crianças prematuras. ${ }^{4,5}$ Sua incidência média no Brasil para recém-nascidos $(\mathrm{RN})$ com peso ao nascimento inferior a $1.500 \mathrm{~g}$ é de $18,4 \%$, sendo inversamente proporcional à idade gestacional e peso ao nascer. ${ }^{6}$

De etiologia multifatorial, acredita-se que além da imaturidade pulmonar e necessidade de ventilação mecânica (VM) e/ou oxigenioterapia, fatores como a sepse, corioamnionite, persistência do canal arterial, enterocolite necrotizante e doença hipertensiva específica da gestação atuem de forma aditiva sobre o pulmão imaturo, gerando inflamação e lesão pulmonar. ${ }^{5,7,8,9}$

Com os avanços tecnológicos clínicos e assistências, a apresentação da DBP se modificou no decorrer dos anos, e a forma clássica e mais severa, com importante fibrose do parênquima, se tornou menos frequente..$^{8,10}$ Atualmente, prematuros extremos que necessitam de ventilação mecânica com baixas pressões e concentrações de oxigênio, adquirem outra forma de DBP, referida como "nova DBP". ${ }^{11}$ Esta aparece como uma manifestação menos agressiva da doença, caracterizada por desenvolvimento alveolar e vascular incompleto. ${ }^{4,5,12}$

De qualquer forma, após a lesão neonatal, o processo de reparação do tecido pulmonar e das paredes das vias aéreas pode levar a alterações na elasticidade do pulmão, diminuição da complacência pulmonar ${ }^{11}$, aumento da resistência das vias aéreas, limita- ção do fluxo aéreo, aumento do volume residual (VR) e diminuição da capacidade residual funcional (CRF). ${ }^{10,13,14,15}$ Estas alterações podem se normalizar com o crescimento, ou persistir por toda infância até a adolescência, predispondo ao desenvolvimento de doença pulmonar obstrutiva crônica (DPOC) na idade adulta. ${ }^{13}$

Diante do exposto, identificar e compreender as alterações ventilatórias e funcionais de crianças e adolescentes com história de DBP, através da análise da função pulmonar e da capacidade de exercício, proporcionará um maior entendimento da doença e, consequentemente, melhora das estratégias de tratamento e prevenção envolvidas em seu manejo. Sendo assim, o objetivo deste estudo foi descrever e analisar, através de uma revisão sistemática, a função pulmonar e capacidade de exercício de crianças e adolescentes que desenvolveram DBP no período neonatal.

\section{Material e método}

\section{Estratégia de busca para a identificação dos estudos}

Para a elaboração desta revisão foram realizadas buscas sistematizadas em três bases de dados eletrônicas SciELO - Scientific Eletronic Library Online (http://www.scielo.br/ acesso em 26 de janeiro de 2014), PEDro - Physiotherapy Evidence Database (http://www.pedro.org.au acesso em 26 de janeiro de 2014) e MEDLINE via Ovid (http:// ovidsp.tx.ovid.com/sp-3.6.0b/ovidweb.cgi acesso em 27 de janeiro de 2014) e foram selecionados artigos publicados até a data de busca.

Os autores elaboraram uma estratégia de busca sensibilizada original para o MEDLINE (via OVID web) e PEDro, apresentadas na figura 1, devido à ausência deste tipo de publicação sobre o tema. Para a base de dados SciELO foram utilizadas as seguintes palavras-chaves: displasia broncopulmonar, bronchopulmonarydysplasia. (Figura 1) 


\section{Medline (via OVID)}

1. Epidemiologic studies/

2. exp case control studies/

3. exp cohort studies/

4. Case control.tw.

5. (cohortadj (study or studies)).tw.

6. cohort analy\$.tw.

7. (Follow up adj (study or studies)).tw.

8. (observationaladj (study or studies)).tw.

9. Longitudinal.tw.

10. Retrospective.tw.

11. Transversal.tw.

12. or/1-12

13. Letter/

14. Historical article/

15. 13 or 14

16. 12 not 15

17. Bronchopulmonary Dysplasia/

18. Pulmonary dysplasia.tw

19. Infant, Premature, Diseases/

20. Respiratory Distress Syndrome Newborn/

21. Respiratory System Abnormalities/

22. Pulmonary Fibrosis/
23. or/17-22

22. Lung Volume Measurements/

23. Respiratory Function Tests/

24. Pulmonary function test ${ }^{\star} . \mathrm{mp}$.

25. Exercise Test/

26. Exercise Tolerance/

27. Spirometry/

28. Lung capacity

29. Vital Capacity/

30. Airway resistance/

32. Infant, Newborn/

33. Infant, Low Birth Weight/

34. Infant, Premature/

35. Neonate.mp.

36. Child*.mp.

37. Pediatr ${ }^{\star} . \mathrm{mp}$.

38. Adolescent*.mp.

39. School age.mp.

40. Child, Preschool/

41. or/32-40

42. 16 and 23 and 31 and 41

\section{PEDro}

\section{Estratégia 1}

Campo Title/abstract: Bronchopulmonarydysplasia

Campo Therapy: respiratory therapy

Campo Method: clinical trial

Campo Problem: No appropriate

\section{Estratégia 2}

Campo Title/abstract: Bronchopulmonary dysplasia

Campo Therapy: respiratory therapy

Campo Method: clinical trial

Campo Problem: impairedventilation

\section{ScIELO}

Displasia broncopumonar ORDoença Pulmonar Crônica Neonatal

Bronchopulmonary Dysplasia OR Neonatal Chronic Lung Disease[all indexes]

Figura 1: Estratégias das bases de dados. 


\section{Critérios para inclusão dos estudos}

Foram considerados critérios de inclusão, estudos que avaliaram tanto a capacidade de exercício quanto a função pulmonar de crianças e adolescentes em idade escolar (6 a 14 anos) com história de DBP no período neonatal. Para a inclusão o diagnóstico de DBP foi definido como a necessidade de oxigênio suplementar acima de $21 \%$ por 28 dias ou mais. ${ }^{9}$ Foram excluídos ensaios clínicos e estudos que examinaram apenas um dos desfechos isoladamente. Também não foram considerados estudos que avaliaram crianças com história de prematuridade extrema onde, a quantidade de crianças que desenvolveram DBP foi explicitada, porém as análises estatísticas não foram realizadas com um grupo DBP distinto, fato que inviabiliza a análise dos efeitos isolados da DBP na função pulmonar e capacidade de exercício nos escolares.

\section{Seleção do material}

O método de triagem dos estudos envolveu 2 examinadores, que analisaram os resultados da pesquisa de forma independente, na busca de estudos potencialmente elegíveis. Foram selecionados os trabalhos que respeitaram os critérios pré-estabelecidos, sendo discutidos os casos duvidosos. Inicialmente os títulos identificados foram armazenados em arquivos, separadamente, de acordo com a base de dados de origem. Na sequência, foi conduzida uma análise por títulos. Com base nos títulos elencados consensualmente, os avaliadores resgataram os resumos e fizeram uma segunda análise, quanto ao conteúdo compatível com os critérios exigidos. Os estudos finalmente eleitos tiveram seus manuscritos adquiridos na íntegra, para estruturação dessa revisão.

\section{Resultados}

Através das estratégias de busca e dos critérios de inclusão estabelecidos para a corrente revisão, foram encontrados um total de 376 estudos. Deste total, após a triagem dos títulos 44 foram selecionados para a análise de seus resumos. Em seguida, 23 artigos foram elencados para a leitura na íntegra, sendo 18 foram rejeitados por não se enquadrarem aos critérios de inclusão. A amostra final foi composta de 5 dos 376 artigos inicialmente identificados (Figura 2).

Grande parte dos estudos apresentados nesta revisão ${ }^{16-19}$ indicam que crianças sobreviventes da
DBP em idade escolar apresentam obstrução variável ao fluxo aéreo com valores de volume expiratório forçado no primeiro segundo $\left(\mathrm{VEF}_{1}\right)$, relação volume expiratório forçado no primeiro segundo por capacidade vital forçada $\left(\mathrm{VEF}_{1} / \mathrm{CVF}\right)$ e fluxo expiratório forçado médio na faixa intermediária da CVF $\left(\mathrm{FEF}_{25-75 \%}\right)$ reduzidos significativamente quando comparados a escolares nascidos a termo que não desenvolveram doença respiratória no período neonatal.

Ao avaliar tanto os escolares com histórico de DBP como aqueles que nasceram prematuramente e não desenvolveram a doença e compara-los a crianças nascidas a termo diversos autores ${ }^{18,20,21}$ identificaram que escolares ex-prematuros também apresentam obstrução ao fluxo expiratório, contudo, a redução dos valores espirométricos é mais amena do que observado nos escolares broncodisplásicos.

Com relação à capacidade a capacidade de exercício os resultados apresentados ainda são controversos. Algumas pesquisas demonstraram similar pico de consumo de oxigênio (VO2pico) entre escolares com DBP e nascidos a termo ${ }^{16,21}$, já outras ${ }^{17,19}$ observaram um menor VO2pico nos escolares com DBP quando comparados aos seus controles nascidos a termo.

A descrição completa dos estudos inclusos nesta revisão e seus resultados estão apresentados na tabela 1 .

\section{Discussão}

Sabe-se que crianças que nasceram prematuras e desenvolveram DBP no período neonatal apresentam até o terceiro ano de idade, disfunção pulmonar com importante limitação do fluxo expiratório, diminuição do $\mathrm{VEF}_{1}$ e do $\mathrm{FEF}_{25-75 \%}$, além de aumento da CRF e VR que caracteriza o aprisionamento de ar. ${ }^{22,23,24}$

Estas alterações acentuadas da função pulmonar durante a primeira infância podem ser amenizadas com o crescimento e desenvolvimento pulmonar, ou persistir por toda infância, adolescência até a idade adulta. ${ }^{13}$ Levando em consideração que o maior crescimento alveolar ocorre durante os primeiros dois anos de vida ${ }^{25}$, é fundamental a manutenção da integridade do sistema respiratório nessa fase. O que vai determinar a melhora da troca de gases e da reserva respiratória após esse período. ${ }^{26} \mathrm{Nessa}$ linha, Balinotti et al. ${ }^{27}$ observaram que bebês nascidos prematuros e com história de doença pulmonar crônica, apresentam diminuição da capacidade de difusão pulmonar 


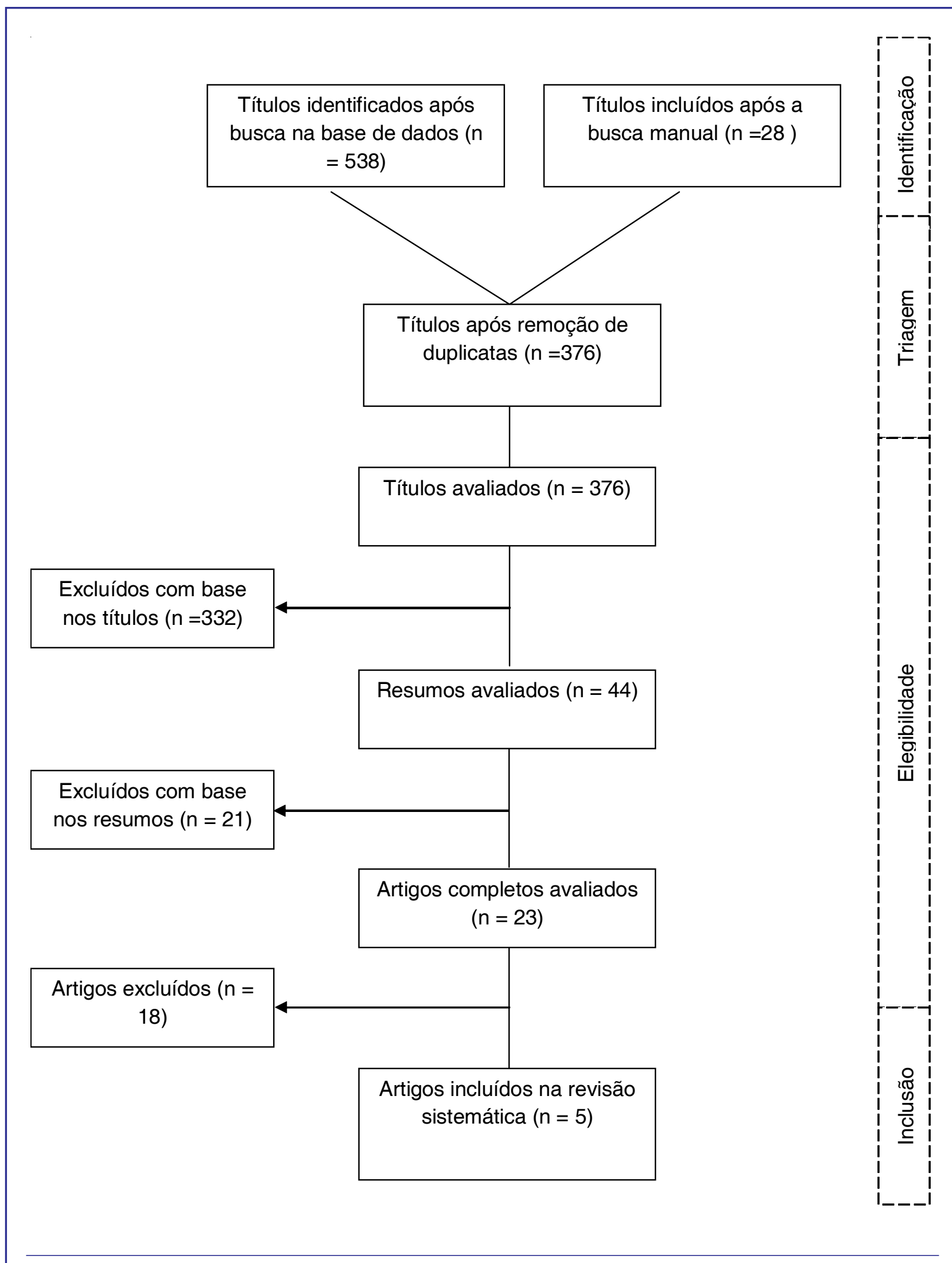

Figura 2: Fluxograma da seleção dos artigos 


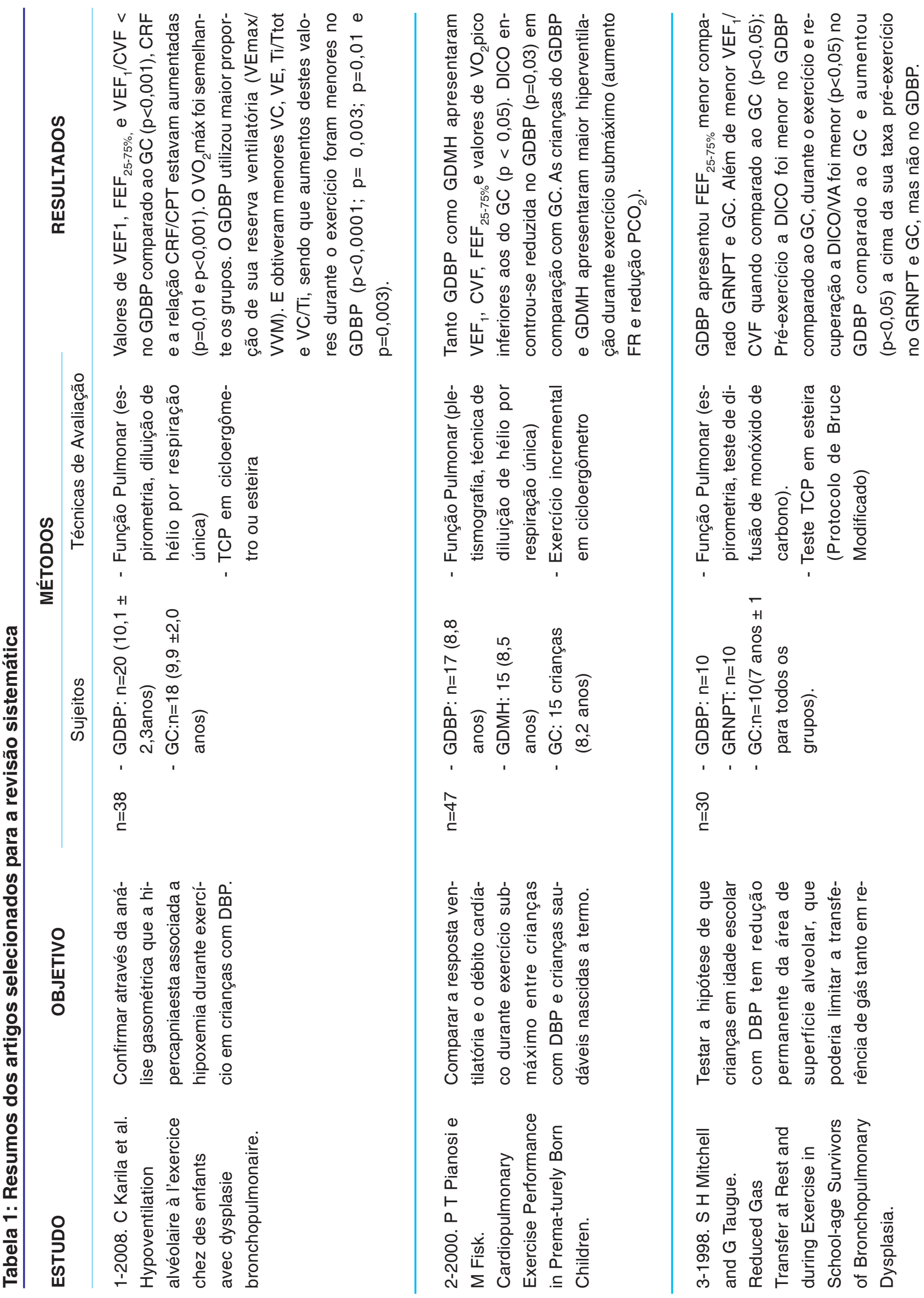




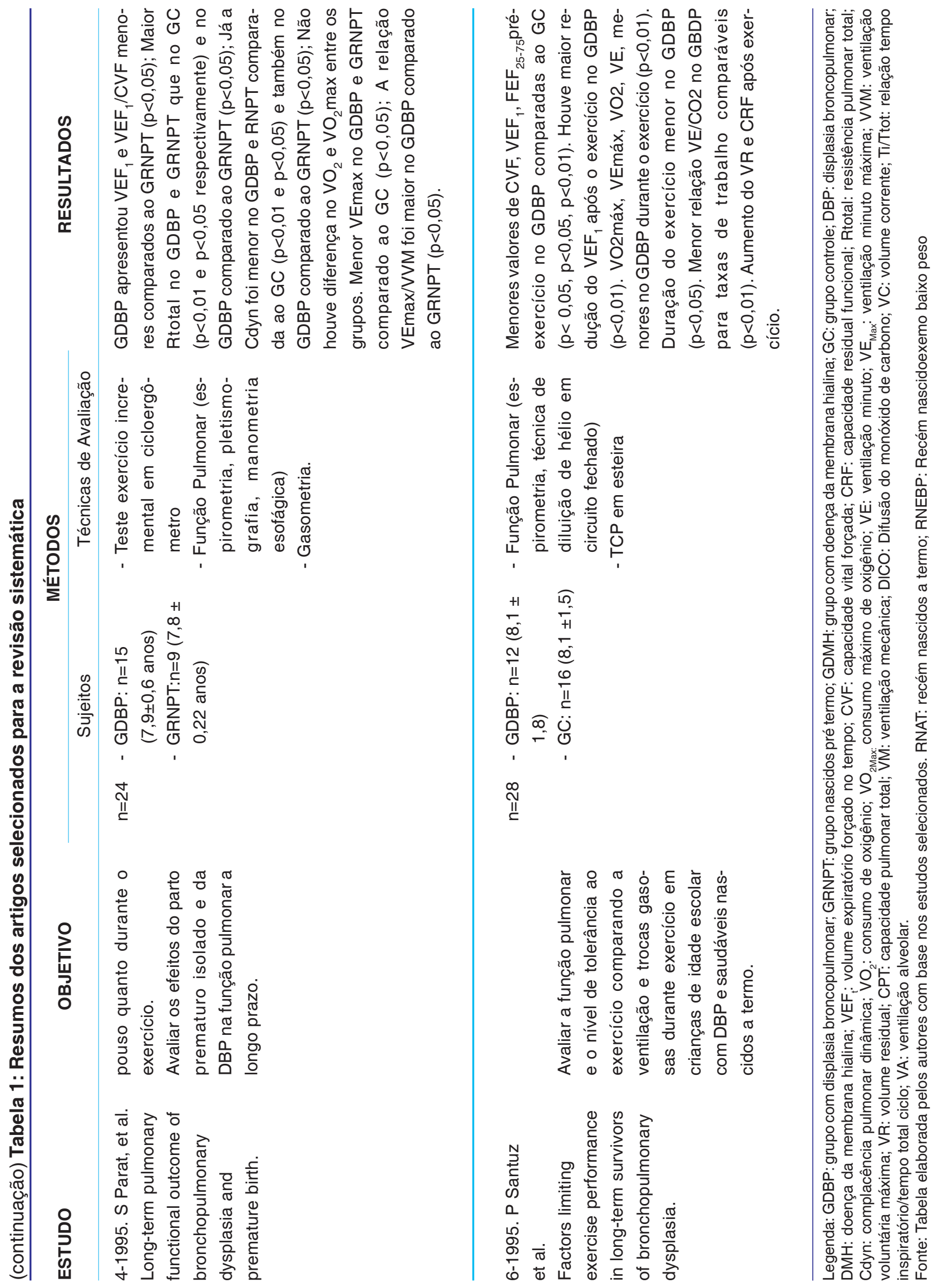


(DlCO), quando comparados aos nascidos a termo. O que indica prejuízo no crescimento alveolar e vascular decorrente da prematuridade associada à pneumopatia.

Já ao avaliar crianças com DBP na idade escolar e compara-las a controles nascidos a termo Pianosi e Fisk ${ }^{17}$, em estudo apresentado nesta revisão, observaram que a difusão de monóxido de carbono está reduzida, porém sutilmente, no grupo DBP. Para os autores, estes resultados sugerem que o leito vascular pulmonar se desenvolveu o suficiente para permitir o recrutamento e acomodar o aumento do débito cardíaco ocasionado pelo exercício.

Com relação ao desenvolvimento ou não da DBP na prematuridade, Vom Hove et. al. ${ }^{28}$ avaliaram separadamente a troca gasosa em escolares que nasceram prematuros com muito baixo peso (PMBP) e não desenvolveram a nova forma de DBP com aqueles que desenvolveram a doença, e observaram que não há diferenças na DICO entre os grupos. Porém, há comprometimento pulmonar com redução de importantes de parâmetros da função pulmonar $\left(\mathrm{VEF}_{1}\right.$, $\left.\mathrm{CVF}, \mathrm{FEF}_{50}\right)$, além de significativamente mais sintomas e tratamentos de asma nos escolares que desenvolveram DBP quando comparados aos que não desenvolveram a doença.

Grande parte dos estudos apresentados nesta revisão ${ }^{16-19}$ conseguiram evidenciar que escolares sobreviventes da DBP apresentam obstrução variável ao fluxo aéreo com valores de $\mathrm{VEF}_{1}, \mathrm{VEF}_{1} / \mathrm{CVF}$, $\mathrm{FEF}_{25-75 \%}$ reduzidos significativamente quando comparados a crianças saudáveis da mesma faixa etária nascidas a termo. Entretanto, o desenvolvimento de DBP no período neonatal parece não ser o único fator que influencia o prejuízo da função pulmonar nestes escolares. Ao avaliar tanto os escolares com histórico de DBP como aqueles que nasceram prematuramente e não desenvolveram a doença e compara-los a crianças nascidas a termo diversos autores ${ }^{18,20,21}$ identificaram que a prematuridade por si só predispõe a obstrução do fluxo expiratório, contudo, a redução dos valores espirométricos é mais menor do que observado nos broncodisplásicos.

Reforçando ainda mais esta evidência, um estudo polonês realizado recentemente avaliou escolares ex-prematuros com e sem DBP, e nascidos a termo e não encontraram diferenças significativas entre os parâmetros da espirometria em prematuros com e sem DBP. Todos os parâmetros investigados foram significativamente inferiores em ambos os grupos (prematuros com e sem DBP) em comparação com os controles a termo, com a exceção do volume de reserva expiratório (VRE\%) que foi significativamente maior. ${ }^{29} \mathrm{O}$ estudo de Santuz et. al. ${ }^{19}$ apresentado nesta revisão, também aponta um aumento do VRE e da CRF nos escolares com DBP, o que indica hiperinsuflação pulmonar e dificuldade no esvaziamento de ar, principalmente durante o exercício. ${ }^{19}$

É relevante relembrarmos que durante a atividade física, o incremento das necessidades metabólicas aumenta a ventilação pulmonar pelo aumento do volume minuto ( $\mathrm{VE}, \mathrm{VE}=\mathrm{VC} \times \mathrm{FR}$ ), qual depende o volume corrente gerado (VC) e da frequência respiratória (FR), e desta forma o coeficiente respiratório se mantem. Diferentemente dos adultos, nas crianças o aumento do VE se ocorre principalmente as custas da elevação na FR entretanto, durante o desenvolvimento da criança até a maturidade a FR tende a cair o $\mathrm{VC}$ aumentar durante o exercício. ${ }^{30}$

Segundo os estudos aqui apresentados na presente revisão, escolares que nasceram prematuros, principalmente os que desenvolveram DBP no período neonatal, apresentam menores valores $\mathrm{VE}$, $\mathrm{VE}_{\mathrm{MÁX}}, \mathrm{VC}^{16,19,21}$, VO2 e VO2 ${ }_{\text {MÁX }}{ }^{17,19}$ e maiores elevações na FR durante o exercício ${ }^{17}$, quando comparados ao grupo de crianças saudáveis nascidas a termo. Outros estudos que avaliaram ex-prematuros, nos quais crianças com DBP foram incluídas, também relatam que a capacidade de exercício expressa pelo VO2 aparece reduzida nesta população. ${ }^{31,32}$ Smith et al. ${ }^{32}$ encontraram uma redução média do $\mathrm{VO} 2$ de 3,9 (IC 95\%: 2,6; 5,2) $\mathrm{ml} / \mathrm{kg} / \mathrm{min}$ em escolares (idade média de 10 anos) que nasceram com $<1000 \mathrm{~g}$, em comparação com os controles a termo. Em um estudo com crianças (média de idade 11 anos) que nasceram com $<26$ semanas de gestação e seus controles, Welsh et al. ${ }^{33}$ descrevem uma redução média do VO2 de 253 (95\% CI: 147, 359) $\mathrm{ml} / \mathrm{min}$ no grupo extremo prematuro, após o ajuste para o peso corporal.

Porém, mesmo tendo o consumo de oxigênio muitas vezes semelhante aos RNPT, crianças com histórico de DBP parecem utilizar uma maior proporção de sua reserva ventilatória (volume minuto máximo / ventilação voluntária máxima $\left.=\mathrm{VE}_{\mathrm{MAX}} / \mathrm{VVM}\right) .{ }^{16,21}$ Jacob et al ${ }^{34}$ verificaram que durante o exercício estes escolares utilizam até $93 \%$ de sua reserva ventilatória, enquanto nas crianças nascidas a termo isso corresponde a 59\%. Este maior uso da reserva ventilatória deve-se provavelmente à redução do número de alvéolos, hiperdistensão alveolar e desordem do 
tecido elástico e fibroso decorrente da doença. ${ }^{22}$ Assim, os escolares broncodisplásicos teriam que superar tanto o aumento da demanda aeróbica quanto o aumento cargas resistivas e elásticas, ocasionando limitação ventilatória durante o exercício nesta população. ${ }^{17}$

No entanto, nem todos estudos demonstram reduções significativas no $\mathrm{VO} 2$ em ex-prematuros com DBP 16,21,35 , assim como em nascidos extremamente prematuros. ${ }^{36,37}$ Abreu et al ${ }^{35}$ ao avaliarem a capacidade cardiopulmonar de escolares brasileiros com história de DBP, RNPT e crianças nascidas a termo, observaram valores do $\mathrm{VO}_{2 \mathrm{MÁx}}$, potência máxima alcançada em relação à potência máxima predita e VE semelhantes entre os grupos, evidenciando serem análogos em relação à aptidão cardiorrespiratória. Já para Zanudin et. al. ${ }^{20}$ a DBP é a única variável perinatal que se relaciona com a aptidão cardiorrespiratória em crianças em idade escolar que nasceram com extremo baixo peso. Em análise de regressão múltipla a DBP consegue explicar $28 \%$ da variação do $\mathrm{VO} 2_{\mathrm{MAX}}$ nesta população.

A menor quantidade de músculo metabolicamente ativo nestas crianças é outra característica pontuada, esse evento foi sugerido por Pianose e Fisk ${ }^{17}$ ao fato de sobreviventes da DBP apresentarem o $\mathrm{VO}_{2 \mathrm{PICO}}$ ligeiramente menor durante exercício submáximo, sendo a massa magra identificada como única variável preditora desse índice. Segundo os autores não há correlação entre a função pulmonar expressa pelo VEF1 e a aptidão cardiorrespiratória expressa pela variável $\mathrm{VO}_{2 \text { PICO. }}$. Da mesma forma, nenhuma das variáveis perinatais como baixo peso ao nascer, idade gestacional, a gravidade da doença da membrana hialina, dias de suporte ventilatório, pressão média de vias aéreas durante $\mathrm{VM}$, ou a razão pressão parcial de oxigênio por fração inspirada de oxigênio $(\mathrm{PaO} 2 /$ FiO2), se relacionou com o com o pico de VO2.

Ao acompanhar uma coorte de adolescentes (18 anos) que nasceram com extrema prematuridade (EP) até a idade adulta (25 anos) Clemm et al. ${ }^{38}$ identificaram que a capacidade de exercício permanece modestamente reduzida no grupo EP, com menores valores de $\mathrm{VO} 2_{\text {PICO }}$ e distância percorrida quando comparados aos controles que nasceram a termo, no entanto dentro dos valores de normalidade. Ainda reforçam que o $\mathrm{VO} 2_{M A ́ x}$ não está relacionado com fatores neonatais e $\mathrm{VEF}_{1}$ atual, mas positivamente associado com atividade física no tempo livre, concordando com os dados concordam com os obtidos por
Pianose e Fisk ${ }^{17}$, assim, a obstrução das vias aéreas em escolares e adultos nascidos prematuros com e sem DBP parece não exercer influência direta na capacidade de exercício.

Nesse contexto, observa-se que a maior parte das pesquisas identificadas sobre o tema foram compostos por amostras em torno de 15 a 28 escolares, apesar dos estudos não apresentarem cálculo amostral. No que diz respeito à idade dos participantes analisados, grande parte avaliou crianças com aproximadamente oito anos, gerando consideráveis evidências em relação à função pulmonar nesta população. Porém, ainda não há evidencias de que as alterações na função pulmonar podem influenciar a capacidade cardiorrespiratória. Em todos os estudos as avaliações foram transversais, não havendo acompanhamento dos indivíduos, a condução de pesquisas longitudinais com esta população viabilizaria o esclarecimento da repercussão dessa doença neonatal sobre a função ventilatória e capacidade de exercício durante o crescimento e desenvolvimento pulmonar.

É importante ressaltar que, nem todos os trabalhos concordam sobre a aptidão cardiorrespiratória em crianças e adolescentes com DBP. As diferenças metodológicas dos estudos dificultam a comparação dos mesmos e a possibilidade de isolar os efeitos da DBP. Alguns trabalhos comparam crianças com DBP e crianças a termo, outros com prematuros sem pneumopatias.

Sabendo que os cuidados durante o período neonatal podem influenciar o prognóstico dos recém nascidos com DBP, e que nos últimos anos a ciência proporcionou importantes avanços na área de cuidados neonatais possibilitando a sobrevida de crianças cada vez mais imaturas, constata-se a necessidade de novas pesquisas que investiguem as implicações da "nova" DBP sobre a função pulmonar e a capacidade de exercício de escolares e a existência de fatores que influenciam diretamente estes resultados a longo prazo, sendo que uma parcela relevante dos estudos analisados nesta revisão avaliaram crianças que nasceram na década de 90.

\section{Consideraçōes finals}

Com base nos achados dessa revisão evidenciou-se que crianças diagnosticadas com DBP no período neonatal apresentam anormalidades na função pulmonar com obstrução variável ao fluxo aéreo durante a idade escolar, estas alterações também apare- 
cem, de forma mais discreta, em escolares expremauros que não desenvolveram DPB. Porém, a função pulmonar expressa pelo $\mathrm{VEF}_{1}$ parece não influenciar o consumo de oxigênio durante o exercício nesta população. Além disso, há aumento do VRE e CRF em escolares com DBP o que predispõe a hiperinsuflação pulmonar, uma possível causa de limitação ventilatória durante o exercício.

Verificou-se uma disposição dos estudos em apontar menor tolerância e ao exercício por estas crianças, sendo que, durante essa prática, estas utilizam uma maior porcentagem de sua reserva respiratória, atingem menores valores de $\mathrm{VE}$ e VC, maiores valores de FR. A capacidade de exercício, expressa pelo VO2 durante o exercício, aparece reduzida em parte dos estudos, porém, esta limitação ao exercício ainda é controversa sendo que algumas pesquisas encontraram VO2pico similares entre escolares que nasceram prematuros com e sem DBP e nascidos a termo.

\section{Referências}

1. Gupta S, Sinhá SK, Donn SM. Ventilatory management and bronchopulmonary dysplasia in preterm infants. Semin Fetal Neonatal Med. 2009; 14: 367-73.

2. Gonzaga AD, Figueira BBD, Sousa JMA, Carvalho WB. Tempo de ventilação mecânica e desenvolvimento de displasia broncopulmonar. Rev Assoc Med Bras. 2007; 53:64-7.

3. Walsh MC, Yao Q, Horbar JD, Carpenter JH, Lee SK, Ohlsson A. Changes in the use of postnatal steroids for bronchopulmonarydys-plasia in 3 large neonatal networks. Pediatrics. 2006; 118:1328-35.

4. Van Marter LJ. Epidemiology of bronchopulmonary dysplasia. Semin Fetal Neonatal Med. 2009;14:358-66.

5. Okamoto CT, Bahr JA, Silva LLG, noronha L. Análises histopatológica e morfométrica no diagnóstico da "nova" displasia broncopulmonar e comparação clinicopatológica com a forma clássica da doença. J Bras Patol Med Lab. 2009; 45: 155-60.

6. Leone CR, Sadeck LSR, Vaz FC, Almeida MFB, Draque CM, Guinsburg R, et al. Brazilian neonatal research network (BNRN): very-lowbirth-weight (VLBW) infant morbidity and mortality. Pediatr Res.2001;49:405.

7. Lima MRO, Andrade MA, Araújo APG, Figueroa JN, Andrade LB. Influência de fatores maternos e neonatais no desenvolvimento da displasia broncopulmonar. Rev Assoc Med Bras.2011; 57:398-403.

8. Bacalari E. Displasia broncopulmonar: um velho problema de cara nova. J Pediatr. 2006;82:2-3.

9. Jobe $\mathrm{AH}$, Bancalari E. Bronchopulmonary dysplasia. Am J Respir Crit Care Med. 2001;163:1723-9.

10. Monte LFV, Silva Filho LVF, Miyoshi MH, Rozov T. Displasia broncopulmonar. J Pediatr. 2005;81:99-100.
11. Vrijlandt EJ, Gerritsen J, Boezen HM, Grevink RG, Duiverman EJ. Lung function and exercise capacity in young adults born prematurely. Am J Respir Crit Care Med. 2006;173:890-6.

12. Coalson JJ. Pathology of new bronchopulmonary dysplasia. Semin Neonatol. 2003; 8: 73-81.

13. Guimarães H, Rocha G, Pissarra S, Guedes MB, Nunes T, Vitor B. Respiratory outcomes and atopy in school-age children who were preterm at birth, with and without bronchopulmonary dysplasia. Clinics. 2011;66:425-30.

14. Brostrom EB, Thunqvist P, Adenfelt G, Borling E, Katz-Salamon $M$. Obstructive lung disease in children with mild to severe BPD. Respir Med. 2010;104:362-70.

15. Perez GP, Merino MN. Displasia broncopulmonar y prematuridad. Evoluciónrespiratoria a corto y a largo plazo. An Pediatr (Barc). 2010;72: 79.e1-79.e16

16. Karila C, Saulnier JP, Elie C, Taupin P, Scheinmann P, Le Bourgeois $\mathrm{M}$, Waernessycle $\mathrm{S}$, Blic J. Hypoventilation alvéolaire à l'exercice chez des enfants avec dysplasiebronchopulmonaire. Rev Mal Respir. 2008; 25:303-12.

17. Pianosi PT, Fisk M. Cardiopulmonary Exercise Performance in Prematurely Born Children. Pediatr Res. 2000;47:653-8.

18. Mitchell SH, Teague WG. Reduced Gas Transfer at Rest and during Exercise in School-age Survivors of Bronchopulmonary Dysplasia. Am J Respir Crit Care Med.1998; 157: 1406-12.

19. Santuz P, Baraldi E, Zaramella P, Filippone M, Zacchello F. Factors limiting exercise performance in long-term survivors of bronchopulmonary dysplasia. Am J Respir Crit Care Med.1995; 152:1284-9.

20. Zanudin A, Gray PH, Burns Y, Danks M, Watter P, Poulsen, L. Perinatal factors in non-disabled ELBW school children and later performance. J Paediatr Child Health. 2013;49: e62-e67.

21. Parat S, Moriette G, Delaperche MF, Escourrou P. Long-term pulmonary functional outcome of bronchopulmonary dysplasia and premature birth. Pediatr Pulmonol. 1995;20:289-96.

22. Robin B, Kim YJ, Huth J, Klocksieben J, Torres M, Tepper RS, Castile RG, Solway J, Hershenson MB, Goldstein-Filbrun A. Pulmonary function in bronchopulmonary dysplasia. Pediatr Pulmonol. 2004;37:236-42.

23. Fakhoury KF, Sellers C, Smith EO, Rama JA, Fan LL. Serial Measurements of Lung Function in a Cohort of Young Children With Bronchopulmonary Dysplasia. Pediatrics. 2010; 125:e1441-7.

24. Talmaciu I, Ren CL, Kolb SM, Hickey E, Panitch HB. Pulmonary function in technology-dependent children 2 years and older with bronchopulmonary dysplasia. Pediatr Pulmonol. 2002;33:181-8.

25. Thurlbeck WM. Postnatal human lung growth. Thorax. 1982; 37:564-71.

26. Bhandari A, McGrath-Morrow S. Long-term pulmonary outcomes of patients with bronchopulmonary dysplasia. Semin Neonatol. 2013;37:132-7.

27. Balinotti JE, Tiller CJ, Llapur CJ, et al. Growth of the lung parenchyma early inlife.Am J Respir Crit Care Med.2009;179: 134-7- a. 
28. Vom Hove M, Prenzel F, Uhlig HH, Robel-Tillig E. Pulmonary outcome in former preterm, very low birth weight children with bronchopulmonary dysplasia: a case-control follow-up at school age. J Pediatr. 2014;146:40-45e4.

29. Konefa ${ }^{3} \mathrm{H}$, Czeszyñska MB, Merritt AT. School-age spirometry in survivors of chronic lung disease of prematurity in the surfactant era. Ginekol Pol. 2013;84:286-292.

30. Rowland TW, Cunningham LN. Development of ventilatory responses to exercise in normal white children. A longitudinal study. Chest. 1997;111:327-32.

31. Kilbride HW, Gelatt MC, Sabath RJ. Pulmonary function and exercise capacity for ELBW survivors in preadolescence: effect of neonatal chronic lung disease. J Pediatr. 2003;143: 488-93

32. Smith LJ, van Asperen PP, McKay KO, Selvadurai H, Fitzgerald DA. Reduced exercise capacity in children born very preterm. Pediatrics. 2008;122:e287e93.

33. Welsh L, Kirkby J, Lum S, et al. The EPICure study: maximal exercise and physical activity in school children born extremely preterm. Thorax. 2010;65:165e72.
34. Jacob SV, Lands LC, Coates AL, Davis GM, MacNeish CF, Hornby L, et al. Exercise ability in survivors of severe bronchopulmonary dysplasia. Am J Respir Crit Care Med. 1997; $155: 1925-9$

35. Abreu LR, Costa-R Angel, RCA, Gastaldi AC, Guimarães RC Ravo SL, Sologuren MJJ. Avaliação da aptidão cardiorrespiratória de crianças com displasia broncopulmonar. Rev Bras Fisioter. 2007;11:105-11.

36. Vrijlandt EJ, Gerritsen J, Boezen HM, Grevink RG, Duiverman EJ. Lung function and exercise capacity in young adults born prematurely. Am J Respir Crit Care Med. 2006;173:890e6.

37. Clemm H, Roksund O, Thorsen E, Eide GE, Markestad T, Halvorsen T. Aerobic capacity and exercise performance in young people born extremely preterm. Pediatrics. 2012;129:e97e105.

38. Clemm HH, Vollsæter M, Røksund OD, Eide GE, Markestad T, Halvorsen T. Exercise Capacity after Extremely Preterm Birth. Development from Adolescence to Adulthood. Ann Am Thorac Soc. 2014;11:537-45. 